\title{
On the Representation of Generalized Analytic Vectors
}

\author{
P. Berglez
}

\begin{abstract}
This paper is concerned with the representation of generalized analytic vectors which are defined as solutions of certain elliptic systems of linear first-order partial differential equations in two complex variables. For the explicite representation of solutions we give integral operators of Vekua type as well as differential operators of Bauer type which map holomorphic vectors onto the set of solutions. For the existence of the differential operators we give a necessary and sufficient condition. We discuss properties of these operators and show how to construct the kernels of the integral operators using the differential operators. Finally we present examples for systems of differential equations for which all the solutions can be represented explicitely.
\end{abstract}

Keywords: Generalized analytic vectors, differential operators, integral operators AMS subject classification: $35 \mathrm{C} \mathrm{05,35} \mathrm{C} \mathrm{10,30} \mathrm{G} \mathrm{20,} 35 \mathrm{~J} 45$

\section{Introduction}

For elliptic systems of differential equations of first order for $2 n$ functions in the plane there exists a far developed theory. In the case $n=1$ the investigations of L. Bers [4] and I. N. Vekua [17] lead to the theory of so called pseudo-analytic and generalized analytic functions. For $n>1$ a wide list of publications deals with the function theoretic properties of the solutions and with boundary value problems (e.g. $[5-7,9-14,20])$. A summary of results and further references can be found in the monographs of $R$. P. Gilbert and J. L. Buchanan [8] and of W. Wendland [19].

Let $\mathbb{N}$ denote the set of natural numbers, $\mathbb{C}$ the set of complex numbers, $\mathcal{D}$ a simply connected domain in the complex plane $\mathbb{C}, \overline{\mathcal{D}}=\{z \in \mathbb{C} \mid \bar{z} \in \mathcal{D}\}, \mathcal{H}(j \times k ; \mathcal{G})(j, k \in \mathbb{N})$ the set of all $(j \times k)$-matrices defined on a domain $\mathcal{G}\left(\mathcal{G} \subseteq \mathbb{C}\right.$ or $\left.\mathcal{G} \subseteq \mathbb{C}^{2}\right)$ the elements of which are analytic functions there, and $E$ the unit matrix.

Here we consider the following system of $j$ linear partial differential equations of first order for a function $w=w(z, \zeta)=\left(w_{1}, \ldots, w_{j}\right)$ of two complex variables $z$ and $\zeta$ in matrix notation

$$
\frac{\partial w}{\partial \zeta}=A(z, \zeta) w+B(z, \zeta) w^{*}
$$

with $A, B \in \mathcal{H}(j \times j ; \mathcal{D} \times \overline{\mathcal{D}})$. Here $w^{*}=w^{*}(\zeta, z)$ denotes the conjugate function to $W=w(z, \zeta)$ in the sense of I. N. Vekua [18: p. 66].

P. Berglez: Technische Universität Graz, Inst. Math., Steyrergasse 30, A - 8010 Graz, Austria

ISSN 0232-2064 / \$2.50 (C) Heldermann Verlag 
Solutions $w \in \mathcal{H}(j \times 1 ; \mathcal{D} \times \overline{\mathcal{D}})$ of this system - which was introduced first by $\mathrm{D}$. Pascali [16] - describe generalized analytic vectors. For conditions under which (1) is the normal form for a $2 n \times 2 n$ real elliptic system see W. Wendland [19: p. $76 \mathrm{ff}$ ].

This paper is concerned with explicite representations for generalized analytic vectors. We prove a general representation theorem giving all the solutions using certain integral operators. Then we define special differential operators which map holomorphic vectors into the set of all the solutions of system (1). We give a necessary and sufficient condition on the coefficients of system (1) for the existence of such differential operators and show how to construct them. These differential operators can also be used to build the kernels of the integral operators introduced.

If $\varphi \in \mathcal{H}(j \times j ; \mathcal{D} \times \overline{\mathcal{D}})$ denotes a non-singular solution of the equation

$$
\varphi_{\zeta}-A \varphi=0
$$

and $w$ an arbitrary solution of equation (1), then the function $v=\varphi^{-1} w$ obeys the equation

$$
v_{\zeta}=c v^{*}
$$

with

$$
c=\varphi^{-1} B \varphi^{*} \in \mathcal{H}(j \times j ; \mathcal{D} \times \overline{\mathcal{D}}) .
$$

Therefore it is sufficient to consider system (2) only.

There is an interesting connection to certain systems of linear differential equations of second order. Every solution $u$ of system (2) where $c$ is assumed to be non-singular satisfies also the system

$$
u_{z \zeta}-c_{z} c^{-1} u_{\zeta}-c c^{*} u=0 .
$$

It can be proved that every solution $v$ of equation (2) can be given in the form

$$
\begin{aligned}
& v=\frac{1}{2}\left(u+\left(c^{*}\right)^{-1} u_{z}^{*}\right) \\
& v=\frac{1}{2 i}\left(u-\left(c^{*}\right)^{-1} u_{z}^{*}\right)
\end{aligned}
$$

where $u$ is a suitable solution of system (3). On the other hand every solution $u$ of system (3) can be represented as

$$
u=v_{1}+i v_{2}
$$

where $v_{1}$ and $v_{2}$ denote arbitrary solutions of equation (2).

As in the scalar case (cf. [17: p. $140 \mathrm{f}$ )) we call the solutions of system (3) complex potentials of equation (2). This connection between the solutions of equation (2) and system (3) will be used to prove a general representation theorem for generalized analytic vectors using differential operators. 


\section{Integral representations after Vekua}

By analogy with the method developed by I. N. Vekua [17: p. $66 \mathrm{ff}]$ which was generalized by $\mathrm{W}$. Wendland for the solution of generalized hypercomplex systems (cf. also [10]) it can be proved that system (2) is equivalent to the integral equation

$$
v(z, \zeta)=\int_{\zeta_{0}}^{\zeta} c(z, \sigma) \int_{z_{0}}^{z} c^{*}(\sigma, s) v(s, \sigma) d s d \sigma+\Phi(z, \zeta)
$$

with

$$
\Phi(z, \zeta)=\int_{\zeta_{0}}^{\zeta} c(z, \sigma) \varphi^{*}(\sigma) d \sigma+\varphi(z)
$$

where $\varphi \in \mathcal{H}(j \times 1 ; \mathcal{D})$ and $z_{0}$ is a fixed point in $\mathcal{D}$ whereas $\zeta_{0}$ is a fixed point in $\overline{\mathcal{D}}$. Equation (5) can be solved iteratively by a Picard process. The solution can be written as

$$
v(z, \zeta)=\Phi(z, \zeta)+\int_{z_{0}}^{z} \int_{\zeta_{0}}^{\zeta} \Gamma(z, \zeta ; \xi, \eta) \Phi(\xi, \eta) d \xi d \eta
$$

Here $\Gamma \in \mathcal{H}(j \times j ; \mathcal{D} \times \overline{\mathcal{D}} \times \mathcal{D} \times \overline{\mathcal{D}})$ denotes the resolvent of the integral equation (5) which itself obeys the integral equations

$$
\begin{aligned}
& \Gamma(z, \zeta ; t, \tau)=c(z, \tau) c^{*}(\tau, t)+\int_{s=t}^{z} \int_{\sigma=\tau}^{\zeta} c(z, \sigma) c^{*}(\sigma, s) \Gamma(s, \sigma ; t, \tau) d s d \sigma \\
& \Gamma(z, \zeta ; t, \tau)=c(z, \tau) c^{*}(\tau, t)+\int_{s=t}^{z} \int_{\sigma=\tau}^{\zeta} \Gamma(z, \zeta ; s, \sigma) c(s, \tau) c^{*}(\tau, t) d s d \sigma .
\end{aligned}
$$

With the aid of the functions

$$
\begin{aligned}
& \Gamma_{1}(z, \zeta ; t, \tau)=\int_{\eta=\tau}^{\zeta} \Gamma(z, \zeta ; t, \eta) d \eta \\
& \Gamma_{2}(z, \zeta ; t, \tau)=c(z, \tau)+\int_{\xi=t}^{z} \int_{\eta=\tau}^{\zeta} \Gamma(z, \zeta ; \xi, \eta) c(\xi, \tau) d \xi d \eta
\end{aligned}
$$

equation (6) can be written as

$$
v(z, \zeta)=\varphi(z)+\int_{\xi=z_{0}}^{z} \Gamma_{1}\left(z, \zeta ; \xi, \zeta_{0}\right) \varphi(\xi) d \xi+\int_{\eta=\zeta_{0}}^{\zeta} \Gamma_{2}\left(z, \zeta ; z_{0}, \eta\right) \varphi^{*}(\eta) d \eta
$$

The functions $\Gamma, \Gamma_{1}$ and $\Gamma_{2}$ are called Vekua resolvents; $\Gamma$ is the principal resolvent and $\Gamma_{1}, \Gamma_{2}$ are the first and second resolvent, respectively.

Now we can establish the following representation theorem using arguments similar to those of Vekua for the scalar case. 
Theorem 1. For every solution $v \in \mathcal{H}(j \times 1 ; \mathcal{D} \times \overline{\mathcal{D}})$ of equation (2) there exists a function $\varphi \in \mathcal{H}(j \times 1 ; \mathcal{D})$ such that for $v$ the representation $(9)$ holds. The function $\varphi$ is then given by

$$
\varphi(z)=v\left(z, \zeta_{0}\right)
$$

On the other hand, every function $\varphi \in \mathcal{H}(j \times 1 ; \mathcal{D})$ generates a solution $v$ of equation (2) by (9).

In order to determine the resolvents needed we investigate some properties of these functions now.

From (7) and (8) it follows

$$
\Gamma(z, \zeta ; t, \tau)=\Gamma_{2}(z, \zeta ; t, \tau) c^{*}(\tau, t)
$$

and from (8) we get

$$
\Gamma_{2}(z, \tau ; t, \tau)=c(z, \tau) \quad \text { and } \quad \Gamma_{2}(t, \zeta ; t, \tau)=c(t, \tau)
$$

Inserting the expression (9) into the equation (2) we get the relations

$$
\begin{aligned}
& \frac{\partial}{\partial \zeta} \Gamma_{1}(z, \zeta ; t, \tau)-c(z, \zeta) \Gamma_{2}^{*}(\zeta, z ; \tau, t)=0 \\
& \frac{\partial}{\partial \zeta} \Gamma_{2}(z, \zeta ; t, \tau)-c(z, \zeta) \Gamma_{1}^{*}(\zeta, z ; \tau, t)=0
\end{aligned}
$$

It can be proved now that the functions

$$
\begin{aligned}
& W_{1}(z, \zeta ; t, \tau)=\frac{1}{2}\left(\Gamma_{1}(z ; \zeta ; t, \tau)+\Gamma_{2}(z, \zeta ; t, \tau)\right) \\
& W_{2}(z, \zeta ; t, \tau)=\frac{1}{2 i}\left(\Gamma_{1}(z, \zeta ; t, \tau)-\Gamma_{2}(z, \zeta ; t, \tau)\right)
\end{aligned}
$$

satisfy equation (2) and obey the conditions

$$
W_{1}(z, \tau ; t, \tau)=\frac{1}{2} c(z, \tau) \quad \text { and } \quad W_{2}(z, \tau ; t ; \tau)=\frac{i}{2} c(z, \tau)
$$

Then $W_{1}$ and $W_{2}$ satisfy the differential equation of the complex potentials (3) also. From (11) it follows that the functions

$$
\begin{aligned}
& \Gamma_{1}(z, \zeta ; t, \tau)=W_{1}(z, \zeta ; t, \tau)+i W_{2}(z, \zeta ; t, \tau) \\
& \Gamma_{2}(z, \zeta ; t, \tau)=W_{1}(z, \zeta ; t, \tau)-i W_{2}(z, \zeta ; t, \tau)
\end{aligned}
$$

are solutions of system (3) also.

In view of (10) we can prove the following 
Lemma 1. Let $w(z, \zeta ; t, \tau)$ be a two-parametric solution of system (3) which satisfies the conditions

$$
w(z, \tau ; t, \tau)=c(z, \tau) \quad \text { and } \quad w(t, \zeta ; t, \tau)=c(t, \tau) .
$$

Then the Vekua resolvents for equation (2) are given by

$$
\begin{aligned}
& \Gamma_{2}(z, \zeta ; t, \tau)=w(z, \zeta ; t, \tau) \\
& \Gamma_{1}(z, \zeta ; t, \tau)=\left(c^{*}(\zeta, z)\right)^{-1}\left[\frac{\partial}{\partial \zeta} \Gamma_{2}(z, \zeta ; t, \tau)\right]^{*} .
\end{aligned}
$$

In order to determine the function $w$ introduced in Lemma 1 we set

$$
w(z, \zeta ; t, \tau)=c(t, \tau) \widehat{w}(z, \zeta ; t, \tau)
$$

Now $\widehat{w}$ has the following properties:

- $\widehat{w}$ is a solution of system (3)

- $\widehat{w}(t, \zeta ; t, \tau)=E$

- $\widehat{w}(z, \tau ; t, \tau)=c^{-1}(t, \tau) c(z, \tau)$.

These conditions define after [18: p. $160 \mathrm{f}]$ the Riemann matrix function $R_{L} \cdot=$ $R_{L} \cdot(z, \zeta ; t, \tau)$ for the system adjoint to system (3) (cf. also [2]). The Riemann matrix function $R_{L}$ for system (3) is then given by $R_{L}(z, \zeta ; t, \tau)=R_{L} \cdot(t, \tau ; z, \zeta)$. With this we have the following relation between the second Vekua resolvent for equation (2) and the Riemann matrix function for system (3).

Lemma 2. Let $R_{L}$ be the Riemann matrix function for system (3). Then

$$
\Gamma_{2}(z, \zeta ; t, \tau)=c(t, \tau) R_{L}(t, \tau ; z, \zeta)
$$

is the second Vekua resolvent for equation (2).

\section{Differential operators after Bauer}

In view of the close relation between the solutions of equation (2) and the complex potentials, which are solutions of system (3) of formally hyperbolic equations, now we turn our attention to systems of linear partial differential equations of second order. In [2] certain differential operators were defined to map holomorphic vectors into the set of solutions of such systems generalizing the operators introduced by $\mathrm{K}$. W. Bauer (cf. [1: Chapter 1]).

Consider the system

$$
w_{z \zeta}+\widehat{A}^{-1} \widehat{A}_{z} w_{\zeta}+\widehat{B} w=0
$$

with $\widehat{A}, \widehat{B} \in \mathcal{H}(j \times j ; \mathcal{D} \times \overline{\mathcal{D}}), \widehat{A}$ non-singular, and the differential operators

$$
K_{n}=\sum_{k=0}^{n} a_{k}(z, \zeta) \frac{\partial^{k}}{\partial z^{k}} \quad \text { and } \quad \tilde{K}_{m}=\sum_{k=0}^{m} b_{k}(z, \zeta) \frac{\partial^{k}}{\partial \zeta^{k}}
$$


with

$$
\begin{aligned}
& a_{k} \in \mathcal{H}(j \times j ; \mathcal{D} \times \overline{\mathcal{D}}) \quad \text { for } k=0, \ldots, n \in \mathbb{N} \cup\{0\} \\
& b_{k} \in \mathcal{H}(j \times j ; \mathcal{D} \times \overline{\mathcal{D}}) \quad \text { for } k=0, \ldots, m \in \mathbb{N} \cup\{0\} .
\end{aligned}
$$

If $a_{n} \not \equiv 0$ in $\mathcal{D} \times \overline{\mathcal{D}}$ or $b_{m} \not \equiv 0$ in $\mathcal{D} \times \overline{\mathcal{D}}$ and if $K_{n} g$ or $\widetilde{K}_{m} h$ is a solution of system (13) in $\mathcal{D} \times \overline{\mathcal{D}}$ for all $g \in \mathcal{H}(j \times 1 ; \mathcal{D})$ or $h \in \mathcal{H}(j \times 1 ; \overline{\mathcal{D}})$, then we call $K_{n}$ a Bauer matrix operator of the first kind of equation (13) in $\mathcal{D} \times \overline{\mathcal{D}}$ (or a $\mathcal{B}_{\mathrm{I}}^{n}$-operator) and $\widetilde{K}_{m}$ a Bauer matrix operator of the second kind of equation (13) in $\mathcal{D} \times \overline{\mathcal{D}}$ (or a $\mathcal{B}_{\mathrm{II}}^{m}$-operator).

In [2] the following theorem concerning the existence of Bauer matrix operators for systems of the form (13) was proved.

Theorem 2. For the system (13) there exists a $\mathcal{B}_{I}^{n}$-operator of the first kind in $\mathcal{D} \times \overline{\mathcal{D}}$ if and only if there is an $n \in \mathbb{N}$ such that with

$$
\begin{aligned}
A_{n} & =\widehat{A} \\
B_{n} & =\widehat{B} \\
A_{k-1}^{-1} A_{k-1, z} & =B_{k}^{-1} A_{k}^{-1} A_{k, z} B_{k}+B_{k}^{-1} B_{k, z} \quad(k=n, \ldots, 1) \\
B_{k-1} & =B_{k}+\left(A_{k-1}^{-1} A_{k-1, z}\right)_{\zeta}
\end{aligned}
$$

we have

$$
B_{0} \equiv 0 \quad \text { in } \quad \mathcal{D} \times \overline{\mathcal{D}}
$$

Then the opererator $K_{n}$ is given by

$$
K_{n}=F_{n-1} \cdots F_{0} \quad \text { with } F_{k}=\frac{\partial}{\partial z}+A_{k}^{-1} A_{k, z} .
$$

Now we consider the differential equation of the complex potentials (3). According to Theorem 2 there exists a $\mathcal{B}_{I}^{n}$-operator for (3) if and only if with

$$
A_{n}=c^{-1} \quad \text { and } \quad B_{n}=-c c^{*}
$$

and the relations (14) the condition (15) is satisfied. By the transformation $\widetilde{w}=c^{-1} w$ we get from (3) the system

$$
\tilde{w}_{z \zeta}+\tilde{A}^{-1} \tilde{A}_{\zeta} \tilde{w}_{z}+\widetilde{B} \tilde{w}=0
$$

with $\tilde{A}=c$ and $\widetilde{B}=c^{-1}\left(\left(c_{z} c^{-1}\right)_{\zeta}-c c^{*}\right) c$. In view of $\widetilde{B}=B_{n-1}^{*}$ and $\widetilde{A}^{-1} \tilde{A}_{\zeta}=$ $\left(A_{n-1}^{-1} A_{n-1, z}\right)^{*}$ and using $\widetilde{B}_{n-1}=\widetilde{B}$ and $\tilde{A}_{n-1}^{-1} \tilde{A}_{n-1, \zeta}=\tilde{A}^{-1} \widetilde{A}_{\zeta}$ we have

$$
\begin{aligned}
\tilde{B}_{n-k} & =B_{n-k}^{*} \\
\widetilde{A}_{n-k}^{-1} \tilde{A}_{n-k, \zeta} & =\left(A_{n-k}^{-1} A_{n-k, z}\right)^{*} \quad(k=1, \ldots, n) .
\end{aligned}
$$

In regard to $(14)$ and (15) it follows $\tilde{B}_{0} \equiv 0$ in $\mathcal{D} \times \overline{\mathcal{D}}$. Therefore the existence of a $\mathcal{B}_{\mathrm{II}}^{m}$-operator $\widetilde{K}_{n-1}$ for system (18) in the form

$$
\widetilde{K}_{n-1}=\tilde{F}_{n-2} \cdots \tilde{F}_{0} \quad \text { with } \quad \widetilde{F}_{k}=\frac{\partial}{\partial \zeta}+\left(A_{k}^{*}\right)^{-1} A_{k, \zeta}^{*}
$$


is ensured:

Now all the solutions of system (3) can be represented as

$$
w(z, \zeta)=K_{n} g(z)+c(z, \zeta) \widetilde{K}_{n-1} h(\zeta)=K_{n} g(z)+c(z, \zeta)\left(K_{n-1} h^{*}(z)\right)^{*} .
$$

(see [2: Theorem 3.3]). Using $G_{n}=-B_{n}^{-1} \partial / \partial \zeta$ and [2: Lemma 2.2] we see that the composition $G_{n} F_{n-1}$ of the two operators $G_{n}$ and $F_{n-1}$ acting on a solution of the equation

$$
\omega_{z \zeta}+A_{n-1}^{-1} A_{n-1, z} \omega_{\zeta}+B_{n-1} \omega=0
$$

gives the identity mapping. As the term $\omega=K_{n-1} h^{*}(z)$ is such a solution we get for the solution $w$ of system (3) the representation

$$
w(z, \zeta)=K_{n} g(z)+\left(c^{*}(\zeta, z)\right)^{-1} \frac{\partial}{\partial z}\left(K_{n} h^{*}(z)\right)^{*}
$$

and the following theorem holds.

Theorem 3. Suppose for system (3) there exists a $\mathcal{B}_{1}^{n}$-operator $K_{n}$ of form (16). Then the following statements are true.

a) For system (3) there exists a Bauer matrix operator of second kind $\widetilde{K}_{n-1}$ also and it is given by

$$
\tilde{K}_{n-1}=c\left(F_{n-2} \cdots F_{0}\right)^{*}=\left(c^{*}\right)^{-1} \frac{\partial}{\partial z}\left(K_{n}\right)^{*}
$$

b) For every solution $w \in \mathcal{H}(j \times 1 ; \mathcal{D} \times \overline{\mathcal{D}})$ of system (3) there exist two holomorphic vectors $g$ and $h$ such that (19) and (20) hold.

c) Conversely, for arbitrary holomorphic vectors $g$ and $h$ the expressions in (19) and (20) are solutions of system (3) in $\mathcal{D} \times \overline{\mathcal{D}}$.

In order to achieve a representation for the solution $v$ of equation (2) we use the first relation in (4) and choose for the solution $u$ of system (3) the expression (19). Thus we have

$$
v(z, \zeta)=\frac{1}{2} K_{n}\left(g(z)+h^{*}(z)\right)+\frac{1}{2} c(z, \zeta)\left(K_{n-1}\left(g(z)+h^{*}(z)\right)\right)
$$

which can be converted into

$$
v(z, \zeta)=K_{n} f(z)+c(z, \zeta)\left(K_{n-1} f(z)\right)^{*}
$$

using $g+h^{*}=2 f$. Therefore the following theorem holds.

Theorem 4. For the solutions of equation $v_{\zeta}=c v^{*}$ defined in $\mathcal{D} \times \overline{\mathcal{D}}$ there exists a representation using Bauer matrix operators if and only if with

$$
B_{n}=-c c^{*} \quad \text { and } A_{n}^{-1} A_{n, z}=-c_{z} c^{-1}
$$

and relations (14) the condition $B_{0} \equiv 0$ in $\mathcal{D} \times \overline{\mathcal{D}}$ holds.

For $f \in \mathcal{H}(j \times 1 ; \mathcal{D})$ the functions

$$
\begin{aligned}
& v=K_{n} f+c\left(K_{n-1} f\right)^{*} \\
& v=K_{n} f+\left(c^{*}\right)^{-1} \frac{\partial}{\partial z}\left(K_{n} f\right)^{*}
\end{aligned}
$$

with $K_{n}$ according to (16) represent solutions of equation (2) in $\mathcal{D} \times \overline{\mathcal{D}}$.

Conversely, in this case every solution of equation (2) defined in $\mathcal{D} \times \overline{\mathcal{D}}$ can be represented in the form (21) or (22) with a vector $f$ holomorphic in $\mathcal{D}$. 


\section{Differential operators and integral representations}

In this section we give an interesting connection between the integral operators after Vekua and the differential operators used just above.

In consequence of the relation between the second Vekua resolvent $\Gamma_{2}$ for the equation (2) and the Riemann matrix functions for the system (3) stated in Lemma 2 we can reduce the determination of the Vekua resolvents to the determination of the Riemann matrix function for the corresponding differential equation of the complex potentials.

In the case of existence of a Bauer matrix operator for a system of form (13) the Riemann matrix function can be represented explicitely using this operator (cf. [2: Theorem 3.1]). Summarizing we have the following assertion.

Theorem 5. Suppose for system (3) there exists a $\mathcal{B}_{I}^{n}$-operator $K_{n}$ according to Theorem 3. Then the second Vekua resolvent $\Gamma_{2}$ for the equation $v_{\zeta}=c v^{*}$ is given by

$$
\Gamma_{2}(z, \zeta ; t, \tau)=c(t, \tau) K_{n} E_{n} \cdots E_{1}\left(A_{0}^{-1}(z, \tau) A_{0}(t, \tau)\right)
$$

using the notation

$$
E_{k} \cdot X=\frac{\partial X}{\partial \tau} B_{k}^{-1}(t, \tau) \quad(k=1, \ldots, n)
$$

and the functions $A_{0}, B_{1}, \ldots, B_{n}$ according to (17) and (14). The first Vekua resolvent $\Gamma_{1}$ is then given by

$$
\Gamma_{1}(z, \zeta ; t, \tau)=\int_{\eta=\tau}^{\zeta} \Gamma_{2}(z, \zeta ; t, \eta) c^{*}(\eta, t) d \eta
$$

or by the expression (12).

\section{Examples}

We consider the system

$$
w_{z \zeta}+B w=0 \quad(B \in \mathcal{H}(j \times j ; \mathcal{D} \times \overline{\mathcal{D}}))
$$

of formally hyperbolic equations. We denote by $\mathcal{H}_{R}(j \times k ; \mathcal{G})$ the set of all functions $f \in \mathcal{H}(j \times k ; \mathcal{G})$ for which $f^{*}=f$ holds. Let $p \in \mathcal{H}_{R}(j \times j ; \mathcal{D} \times \overline{\mathcal{D}})$ be a particular, non-singular solution of system (23) and $w \in \mathcal{H}_{R}(j \times 1 ; \mathcal{D} \times \overline{\mathcal{D}})$ an arbitrary solution of system (23). After [3: Theorem 1] the function $v=p^{-1} w$ represents a solution of the system

$$
v_{z \zeta}+p^{-1} p_{\zeta} v_{z}+p^{-1} \dot{p}_{z} v_{\zeta}=0
$$

If $V \in \mathcal{H}_{R}(j \times 1 ; \mathcal{D} \times \overline{\mathcal{D}})$ is a solution of system (24), then $u=V_{z}$ is a solution of the system

$$
u_{\zeta}=-p^{-1} p_{\zeta} u-p^{-1} p_{z} u^{*}
$$


Transforming according to $u=p^{-1} U$ the function $U$ satisfies the system

$$
U_{\zeta}=-p_{z} p^{-1} U^{*} \text {. }
$$

The differential equation of the complex potentials to system (25) runs

$$
\hat{u}_{z \zeta}+\left(p_{z} p^{-1}-p_{z z} p_{z}^{-1}\right) \hat{u}_{\zeta}-p_{z} p^{-1} p_{\zeta} p^{-1} \hat{u}=0 .
$$

A solution of this system is given by

$$
\hat{u}=p \frac{\partial}{\partial z}\left(p^{-1} W\right)
$$

where $W \in \mathcal{H}_{R}(j \times 1 ; \mathcal{D} \times \overline{\mathcal{D}})$ denotes an arbitrary solution of system (23). Now if a representation of the solution of system (23) using differential operators is known, the solution of system (25) can be given by differential operators also.

Especially, for the matrix $B=\left(B_{\text {rs }}\right) \in \mathcal{H}_{R}(j \times j ; \mathcal{D} \times \overline{\mathcal{D}})$ given by

$$
B_{r s}= \begin{cases}-\frac{n(n+1)}{\eta^{2}} & \text { for } r=s, 1 \leq s \leq j \\ -1 & \text { for } r=s+1,1 \leq s \leq j-1 \\ 0 & \text { for } r \neq s, s+1\end{cases}
$$

with $\eta=z+\zeta$, all solutions of system (23) can be given for $n \geq j-1$ with the aid of the Bauer matrix operator $K_{n}$ as

$$
w=K_{n} g+\left(K_{n} h^{*}\right)^{*}
$$

with

$$
\begin{aligned}
& K_{n}=\sum_{k=0}^{n} a_{k} \frac{\partial^{k}}{\partial z^{k}}, \quad a_{k}=\left(a_{k, r s}\right) \in \mathcal{H}_{R}(j \times j ; \mathcal{D} \times \overline{\mathcal{D}}) \\
& a_{k, r s}= \begin{cases}\frac{(-1)^{n-k-r+s}(2(n-r+s)-k) !}{(r-s) ! k !(n-r+s-k) ! \eta^{n-k-2 r+2 s}} & \text { for } 0 \leq r-s \leq n-k \\
0 & \text { otherwise }\end{cases} \\
& g \in \mathcal{H}(j \times 1 ; \mathcal{D}), \quad h \in \mathcal{H}(j \times 1 ; \overline{\mathcal{D}})
\end{aligned}
$$

(cf. [3]). Then the solutions $w_{R} \in \mathcal{H}_{R}(j \times 1 ; \mathcal{D} \times \overline{\mathcal{D}})$ of system (23) have the form

$$
w_{R}=K_{n} g+\left(K_{n} g\right)^{*} \quad \text { with } g \in \mathcal{H}(j \times 1 ; \mathcal{D}) \text {. }
$$

With a particular solution $p \in \mathcal{H}_{R}(j \times j ; \mathcal{D} \times \overline{\mathcal{D}})$ of system (23) we get for the solution $U$ of system (25)

$$
U=p \frac{\partial}{\partial z}\left(p^{-1} w_{R}\right)
$$

For example, the function $p=\left(p_{r s}\right) \in \mathcal{H}_{R}(j \times j ; \mathcal{D} \times \overline{\mathcal{D}})$ with

$$
p_{r s}=\left\{\begin{array}{ll}
\frac{(-1)^{n-r+s}(2(n-r+s)) !}{(r-s) !(n-r+s) ! \eta^{n-2 r+2 s}} & \text { for } r \geq s \\
0 & \text { for } r<s
\end{array} .\right.
$$

is a suitable particular solution of system (23). 


\section{References}

[1] Bauer, K. W. and St. Ruscheweyh: Differential Operators for Partial Differential Equations and Function Theoretic Applicatons. Lect. Notes Math. 791 (1980), $1-258$.

[2] Berglez, P.: Differential operators for systems of linear partial differential equations. Ann. Mat. Pura Appl. 162 (1992), 263 - 279.

[3] Berglez, P.: On the representation of solutions of systems of linear partial differential equations (to appear).

[4] Bers, L.: Theory of Pseudo-Analytic Functions. New York: New York University 1953.

[5] Bojarski, B.: Theory of generalized analytic vectors (in Russian). Ann. Polon. Math. 17 (1966), $281 \cdot 320$.

[6] Buchanan, J. L.: A similarity principle for Pascali systems. Complex Variables 1 (1983), $155-165$.

[7] Douglis, A.: A function-theoretic approach to elliptic systems of equations in two variables. Comm. Pure Appl. Math. 6 (1953), 259 - 289.

[8] Gilbert, R. P. and G. Hile: Generalized hypercomplex function theory. Trans. Amer. Math. Soc. 195 (1974), 1 - 29.

[9] Gilbert, R. P. and J. L. Buchanan: First Order Elliptic Systems: A Function Theoretic Approach. New York: Acad. Press 1983.

[10] Gilbert, R. P. and W. Wendland: Analytic, generalized, hyperanalytic function theory and an application to elasticity. Proc. Roy. Soc. Edinburgh (Sect. A) 73 (1975), 317 - 331.

[11] Goldschmidt, B.: Funktionentheoretische Eigenschaften verallgemeinerter analytischer Vektoren. Math. Nachr. 90 (1979), 57 - 90.

[12] Goldschmidt, B.: A maximum principle for generalized analytic veciors. Applicable Anal. 12 (1981), 139 - 152.

[13) Goldschmidt, B.: Regularity properties of generalized analytic vectors in $\mathbb{R}^{n}$. Math. Nachr. 103 (1981), 245 - 254.

[14] Hile, G. N.: Representations of solutions of a special class of first order systems. J. Diff. Equ. 25 (1977), 410 - 424.

[15] Muskhelishvili, N. I.: Singuläre Integralgleichungen. Berlin: Akademie-Verlag 1965.

[16] Pascali, D.: Vecteurs analytiques généralisés. Rev. Roumaine Math. Pures Appl. 10 (1965), 779 - 808.

[17] Vekua, I. N.: Verallgemeinerte analytische Funktionen. Berlin: Akademie-Verlag 1963.

[18] Vekua, I. N.: New Methods for Solving Elliptic Equations. Amsterdam: North Holland Publ. Comp. 1967.

[19] Wendland, W.: Elliptic Systems in the Plane. London: Pitman 1979.

[20] Youvaraj, G. P. and R. K. Jain: On pseudo-analytic matrix functions. Complex Variables 15 (1990), 259 - 278. 